\title{
The Role of INTEGRIS in the A2A Reti Elettriche's Roadmap Toward the Smart Grid
}

\author{
D. Della Giustina, S. Zanini, S. Pugliese and L. Cremaschini \\ A2A Reti Elettriche SpA \\ via Lamarmora 230, 25124 Brescia, Italy \\ davide.dellagiustina@a2a.eu
}

\begin{abstract}
A2A Reti Elettriche SpA is an electricity distributor that operates in a large area in the northern part of Italy which includes the cities of Milan and Brescia. The paper describes the main goals that A2A Reti Elettriche has set forth in order to respond to the new challenges in operating electricity grids. More specifically, it examines the 7th Framework Program European Project INTEGRIS which revolves around a hybrid and distributed ICT infrastructure aimed at enabling the new electric functionalities demanded by a Smart Grid model.
\end{abstract}

Keywords-Smart grids; Power distribution; Distributed power generation; Distributed information systems; Networking for the smart grid

\section{INTRODUCTION}

A2A Reti Elettriche SpA [1] (ARL) is a fully owned subsidiary of the A2A group that manages electricity distribution in the cities of Milan and Brescia and in 46 other municipalities in the northern part of Italy. ARL serves over 1.1 million customers and distributes over $11 \mathrm{TWh}$ of energy per annum. ARL grid is spread across both highly dense urban areas as well as extra-urban, lacustrine and mountainous environments - which therefore present diverse characteristics and issues.

ARL electric distribution operates in a national context characterized by regulatory pressures aimed at improving the continuity of service, by progressively reducing number and length of disconnections per user as defined by the Italian Authority of Electrical Energy and Gas (AEEG) in [2]. AEEG is also considering to define new remuneration/penalization mechanisms related to the power quality starting from 2012 [3].

The pressures from national authorities are compounded by other pressures prompted by transnational programs, aimed at protecting the environment - such as the European "20-2020 " directive [4] - that are giving impulse to the improvement of the electric system efficiency and intelligent grid management of distributed generation (DG) from renewable, distributed energy storage, electric vehicles (EV), etc.

This radical change will have to deal with other critical aspects stemming from changes in the legislative and economic framework. The unbundling of the energy supply chain, for instance demands a strict separation of duties and roles across the various players: market, transmission, distribution, telecom operator, etc.
The energy market is going to undergo a massive change with the explosion of the number and size of new participants - either end-consumers or micro-producers. New providercustomer (prosumers) commercial models will take hold, with the emerge of new categories of intermediaries and franchisees - interest groups representing associations clustered around local geography (district, precinct, etc.) or vertically integrated around business interests (small offices, etc.), local producers or "aggregators".

Lastly, the information exchange between distribution and transmission operators will have to peak to a new level in terms of volume, complexity and time interval, in order to manage the new topology with several aggregation hubs to ensure energy availability and ancillary services. In other words, the DSO will progressively transform into a dispatcher.

The ongoing transformation of the electric distribution system, described above in the backdrop of the drive to smarter, more intelligent - Smart Grids.

ARL has a goal of continuous efficiency and effectiveness improvement. This goal can only be achieved through a strategy centered on sustainable technological innovation, which translates into a gradual convergence between the electric energy (EE) and the information and communication technologies (ICT) foundations.

For this reason ARL joins the 7th Framework Program European Project INTEGRIS [5] in partnership with eight partners - including research and development, academic and business and coordinated by ENDESA. INTEGRIS aims to development a novel and flexible communication infrastructure based on broadband power line communication (BPL) and high-rate wireless communications system, such as Wi-Fi. The INTEGRIS heterogeneous and meshed communication environment promises to pursue a balanced tradeoff between DSOs' investments/benefits, meeting most of the requirements foreseen for the Smart Grids.

The paper describes - in Section II - how the electric grid of ARL is managed today and which tools are used - in Section III - how ARL plans to transform them and what changes are being envisioned. In Section IV is described the INTEGRIS project architecture as a possible driver to enable the change toward the Smar Grid model. 


\section{STATE OF THE ART OF ARL'S DisTRIBUTION GRID}

The distribution network of ARL is made up by the two isolated grids of Milan and Brescia. Overall it includes 38 $\mathrm{HV} / \mathrm{MV}$ transformer substations (PS), $28 \mathrm{MV}$ dispatching substations and over $8300 \mathrm{MV} / \mathrm{LV}$ transformer substations (SS).

Hereafter are illustrated the technologies used to manage the electric grid of ARL. Those technologies are not consistently deployed all over the Brescia and Milan network, thus this portrait describes an "average" condition of ARL network status.

PSs have been already equipped with Substation Automation Systems (SAS), based nevertheless on proprietary standards from different vendors. SAS consists of intelligent electric devices (IEDs), such us protection relays, which are used to protect assets in primary substation in case a fault occurs, typically opening the main circuit breaker of the MV line.

A supervisory system (SCADA) is used to manage the two areas allow the telecontrol of all $\mathrm{HV} / \mathrm{MV}$ and $\mathrm{MV} / \mathrm{MV}$ substations and of roughly the $20 \%$ of the MV/LV substations. It continuously receives feeds on network states and alarms via remote terminal units (RTUs). RTUs allow the sending of remote command to disconnectors or circuit breaker that alter the topology of the MV grid when is needed.

In recent years, along with SCADA and SAS, ARL has launched an effort to make the $\mathrm{MV} / \mathrm{LV}$ grid network management more pervasive. The energy distribution peaks in summer time, due to the high presence of air cooling appliances, has induced ARL to introduce a $\mathrm{MV} / \mathrm{LV}$ monitoring system (SMS) which now covers about $50 \%$ of the SSs and it is still in deployment phase. Likewise, in all PSs ARL has already installed Power Quality analysis tools (PQS) on MV busbars in order to be ready for the new target set by the Authority.

Some fault location Systems (FLS) such as [6], are getting underway.

Lastly, ARL has now completed the deployment of electronic meters at the edge of customers home and low voltage concentrators (LVC) in SS, and its own Automatic Meter Management system (AMM). However, AMM provides commercial functionalities only (user connectiondisconnection, power curtailment, billing, etc.) and it is not used to manage the grid.

Compared to other European DSOs, ARL's network management state of the art is characterized by a fairly good level of technology penetration. That notwithstanding, the deployment of substation systems has taken place in the 90's and early 2000, when the role of ICT was still less relevant. Therefore the technology introduction was characterized by a proliferation of centralized and vertical subsystems, often not interoperable and hardly underpinned by a coherent communication architectural design (Fig. 1).

From an application architecture stand point, centralized and vertical models translate into:

- $\quad$ each device installed at substation level transfers data from the field to the center and there is no data processing occurring at substation level - with some exceptions for SAS.

- central application subsystems do not exchange information amongst ourselves - making the workflow management at the network management not efficient.

From a communication point of view, even though primary stations are already connected via high speed, high reliability fiber optics (FO) or copper connections, MV network communication infrastructure leaves much to be desired. Each system uses a dedicated GSM communication which causes low efficiency and unnecessary operating costs. The total number of GSM equipments to support the communication with the peripheral devices (LVC for electronic meters, RTUs for both the SCADA and the monitoring system) is reported in TABLE 1.

TABLE 1

\begin{tabular}{|l|r|}
\hline \multicolumn{1}{|c|}{ Device } & GSM (SIM/modem) \\
\hline LVC & 8720 \\
\hline SMS RTU & 3726 \\
\hline SCADA RTU & 1392 \\
\hline & $\mathbf{1 3 8 3 8}$ \\
\hline
\end{tabular}

Regarding the communication with electronic meters, a proprietary narrow band PLC technology is used today. This technology, in terms of performance, is not able to deal with the transition from the present electronic meter to the smart meters, which ARL would use to get a continuous feedback on the LV grid status.

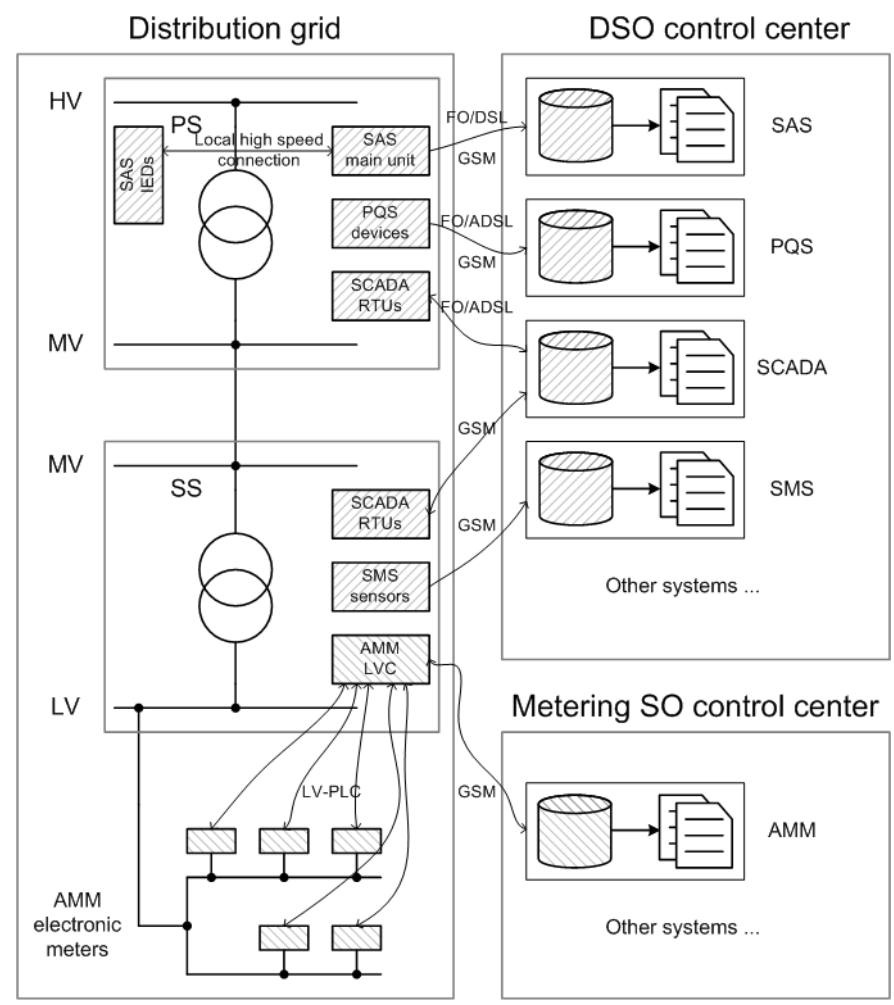

Fig. 1. Schematic depiction describing ARL's grid management systems. It provides an "average" view of Milan and Brescia grids. 
This scenario highlights the priority and the focus on redesigning a modern ICT architecture to support today's and tomorrow's distribution management needs, such as those driven by the changing regulatory framework, that we have briefly described in the introduction, and the integration of distributed energy resources, with a particular focus on DG from renewable energy sources.

\begin{tabular}{|l|l|}
\multicolumn{2}{l}{ TABLE 2} \\
\hline Photovoltaic LV & 2027 sites \\
& $18,9 \mathrm{MWp}$ installed \\
\hline Other LV connections & 14 sites \\
& $1,7 \mathrm{MW}$ \\
\hline MV connections & 92 Sites \\
& $171 \mathrm{MW}$ installed \\
\hline
\end{tabular}

The level of penetration of DG sources into ARL's grid is summed up in TABLE 2. At present, the LV distributed generation is not such as to create much anxiety at operation level - given that the maximum hosting capacity level has not been reached yet. However the DG connection demand is continuously growing, and therefore forcing ARL to put a strong focus and figure out a way to manage the issues.

For the MV, new co-generation (combined heat and electricity) plants, with large production power peak up to 24 MW are being connected to support the development of public district heating, as well as serving hospitals or other business users (e.g. airports). Photovoltaic and micro-hydroelectric plants, in the range of $1-4 \mathrm{MW}$ are also present on the MV grid.

Regarding Electric vehicles, the A2A group - through its emobility initiative in partnership with Renault - is actively involved and plans to deploy a network of public recharging points in the urban areas of Milan and Brescia.

\section{SMART GRID APPLICATION SCENARIO}

The above scenario is pushing ARL to use new functionalities to more effectively manage its grid. Some the most relevant electrical application foreseen for the Smart Grid were the integrated grid monitoring, from primary substations to final consumers and producers, described in [7], and LV network automation functions, such as those involving the interaction with smart metering and home automation, described in [8].

Another essential application, in order to improve the quality of service and exploiting the presence of DG, is the evolution of protection schemes - today serving only at primary substation level - toward extended schemes covering secondary substations, distributed generation attached to MV and capable of implementing block/trip logics.

To better understand the benefit of extending protection systems outside the primary substations, it is worth recalling how today faults are located and isolated in order to restore the service (Fig. 2).

When a fault occurs on a MV line, the feeder protection relay $\left(R_{1}\right)$ in primary substation opens the circuit breaker, sends an alarm to the SCADA system to notify the event, and all the customers under that line are affected by the outage.
Control center operators coordinate the binary research of the fault in cooperation with field crews.

The switch disconnector in the secondary substation located in the middle of the MV line is open, while the control center staff closes the circuit breaker, trying to re-feed the line. If the $R_{1}$ detects again the fault, this means that the fault is located in left part of the MV line, otherwise the fault is in the second half. The process is iterated until the faulted trunk is located. This cable is kept disconnected and the service is restored feeding SSs in stand-by with the other PS.

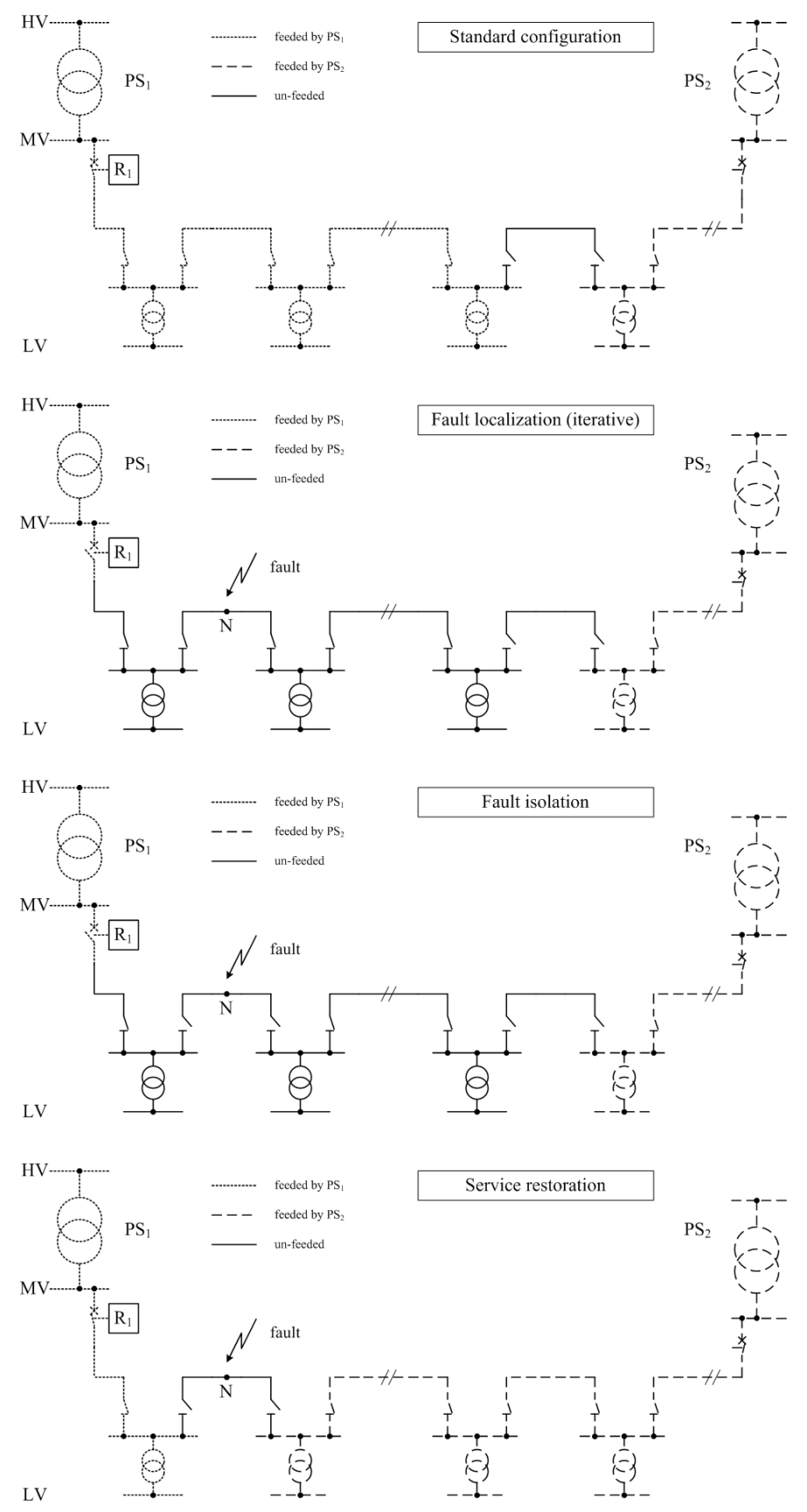

Fig. 2. Fault location, isolation ad service restoration procedure. 
If no switch disconnector can be remotely controlled by the SCADA, this (iterative) process would require up to $1 \mathrm{~h}$, according to the number of SSs involved, their relative distance, the traffic, weather condition, etc. If the switch disconnector in the middle of the line were controllable, the time need would be reduced roughly by a factor 2 . That is the reason why ARL is working to install RTUs over 30\% of the secondary substations.

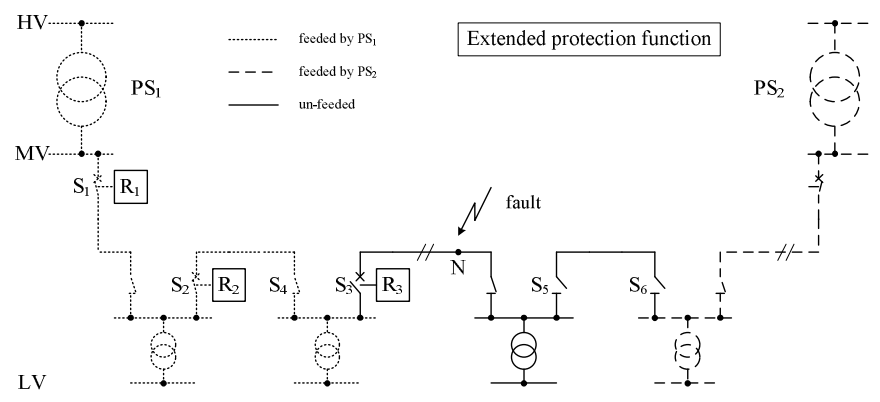

Fig. 3. Basic principle of the extended protection function described in the paper.

A real step forward can be done installing circuit breaker and protection relays in some secondary substation, e.g. in the middle of the line, where a DG is connected to the MV busbar. If so, an extended protection function can be performed. Its basic principle can be explained making reference to the scheme depicted in Fig. 3. When a fault occurs over the MV network, the protection relay closest to the fault $\left(R_{3}\right)$ sends a block message to the other protection relays $\left(R_{1}\right.$ and $\left.R_{2}\right) . R_{3}$ isolates the fault opening the switch $S_{3}$, while relays $R_{1}$ and $R_{2}$ do not open their respective switches maintaining part of the feeder active. If $\mathrm{S}_{4}, \mathrm{~S}_{5}$ and $\mathrm{S}_{6}$ are remotely controlled, the operator at the control center of the utility can issue a command to feed disconnected secondary substations in by $\mathrm{PS}_{2}$.

From a communication standpoint, protection system logic consists in exchanging small messages with an extremely low latency and an extremely high level of reliability. Common values for the transfer time are:
Short circuit
$\leq 20 \mathrm{~ms}$
Ground fault (isolated neutral)
$\leq 200 \mathrm{~ms}$
Ground fault (compensated neutral)
$\leq 900 \mathrm{~ms}$

Commands to change the status of remotely controlled disconnectors and breakers are small messages which have to be transferred in few seconds.

Applications such those described in [5, 6] (integrated monitoring and LV grid management) requires an higher transfer rate to cope with the large amount of data which must be optimized in some way, but they impose less severe requirements in terms of availability and reliability. A comparisons between those electrical function is reported in TABLE 3, which is an extract of the analysis performed by INTEGRIS.
TABLE 3

\begin{tabular}{|l|r|r|}
\hline \multicolumn{1}{|c|}{ Nome } & \multicolumn{1}{|c|}{ Latency } & \multicolumn{1}{c|}{ Reliability } \\
\hline Protection & $\leq 20 \mathrm{~ms}$ & $99,999 \%$ \\
\hline Command & $\leq 2 \mathrm{~s}$ & $99,99 \%$ \\
\hline Monitoring & $\leq 2 \mathrm{~s}$ & $99,99 \%$ \\
\hline
\end{tabular}

ICT is the key enabler to deploy these new functionalities. ARL has chosen to launch a set of initiatives and pilot project - partly funded internally and partly co-funded at European and national level. They aim at:

- acquiring knowledge and skills in this area,

- testing new technologies available on the market,

- $\quad$ supporting the development of new solutions.

Amongst these initiatives, the most important for ARL is the 7th Framework Program European Project INTEGRIS. Within the project scope and roles, ARL has focused its contribution in defining a Smart Grid evolution scenario - the functional and systems requirements; the coordination of multiple field test of the INTEGRIS architecture - over its network and two other field test sites.

The goal of the INTEGRIS project is the design and the test of an ICT architecture enabling the Smart Grids that be:

1. heterogeneous, i.e. integrating multiple communication technologies (BPL, wireless),

2. distributed, i.e. enabling the distribution of "intelligence" across the network,

3. open, i.e. underpinned by open (nonproprietary) standards (e.g. IEC 61850, IEC 62056, ...)

Design points are based on rationales that we describe in this section.

The choice of a hybrid communication infrastructure is without alternative because there is no one single communication technology that possesses the right balance between performance and total cost (including maintenance). In fact a pervasive FO cabling is costly and requires long time to deploy. The solution envisioned is therefore based on a mix of:

- FO where already available or easy to install where overhaul of existing or new cabins are planned,

- BPL over MV cables,

- $\quad$ High rate wireless - more specifically Wi-Fi.

INTEGRIS focuses on BPL and Wi-Fi, as they are easier to deploy over the electric grid. WiMax was also considered as an alternative to $\mathrm{Wi}-\mathrm{Fi}$. It was not included in the project scope as it requires a license in many European countries including Italy. The use of MV-BPL and Wi-Fi is not mutually exclusive (one of the other are used to reach a substation) nor completely mutually inclusive (use of both to reach substation). INTEGRIS rather proposes a low level integration of these two technologies (ISO/OSI model Level 2). This choice allows the improvement of network performance and reliability, whilst containing costs. BPL over LV power cables has been chosen because it provides higher transfer rate with respect to narrow band PLC, both proprietary technologies and open PLC standards such as PRIME. BPL and Wi-Fi provides and Ethernet-like communication, therefore they are 
compatible with the IP protocol. For this reason, this ICT grid can be consider as wide area network superimposed to the EE distribution grid.

The central NMS supervision software, along with advanced algorithms for package routing distributed in the substations, manage the hybrid communication network. Both components are part of the innovation contributed by the INTEGRIS project.

Another aspect in scope is network traffic prioritization, which is demanded by diverse electrical functionalities with diversified latency requirements. Examples are block/trip messages issued by protections that require higher priority versus messages used for monitoring. Notwithstanding the advancements brought about by the use of a hybrid network and the new priority management mechanisms, the high level of performance demanded by active protections (particularly with short circuits) cannot be achieved unconditionally by the hybrid communication architecture. In testing these new functionalities, therefore the INTEGRIS project approach tends to be stochastic rather than deterministic - i.e. determining the percentage of faults resolved over the total number of faults.

From a data management standpoint, the new challenge is the exponential growth of information collected that would have to flow from the periphery of the grid to the higher level, in particular the control center. The solution envisioned consists of a distributed management system across all grid network nodes (control center, primary and secondary substations) thus reducing the unnecessary network traffic and allows to take faster decisions. It consists of a distributed data storage and a distributed data process center, which file and compute locally measurements got from meters and sensors. Each node of the management system provides alarms to higher nodes of the grid in case either faults or faulty conditions occur. The key hub of this distributed network storage system is the secondary (MV/LV) substation - where along with the electric information are located information related to end users from smart meters, up to now only relevant to commercial application subsystems.

Lastly, standardization is extremely relevant at any level and particularly for what pertains EE standards. The coherent and integral grid network management is predicated upon the emergence and integral adoption of standards. Thus INTEGRIS aims at extending the IEC 61850 standard beyond primary substations - reaching secondary substations, for trip/block protection message management as well as typical monitoring related message management. For smart meter messaging INTEGRIS has chosen to adopt the emerging IEC 62056 - DLMS/COSEM protocol. In order to consolidate meter information at secondary substation level an $61850 / 62056$ conversion mechanism is required.

\section{CONCLUSIONS}

ARL is in the process of re-engineering its own ICT infrastructure to support the new advanced functionalities needed to transform the electric grid from today to accommodate tomorrow's needs. The INTEGRIS project deals with a new ICT architecture that incorporates most of ARL requirements and is considered a key milestone along the way to the transformation toward the Smart Grids.

\section{ACKNOWLEDGMENT}

This work was supported in part by EU's seventh framework funding program FP7 (INTEGRIS project ICTEnergy-2009 under grant 247938).

\section{REFERENCES}

[1] A2A Reti Elettriche SpA [Online]. Available: www.a2aretielettriche.eu

[2] AEEG, "ARG/elt 333/07 - Testo integrato della regolazione della qualità dei servizi di distribuzione, misura e vendita dell'energia elettrica per il periodo di regolazione 2008-2011" [Online]. Available: www.autorita.energia.it/it/docs/07/333-07.htm

[3] AEEG, "DCO 42/10 - Regolazione della qualità dei servizi elettrici nel IV periodo di regolazione (2012-2015)" [Online]. Available : www.autorita.energia.it/it/docs/dc/10/042-10dco.jsp

[4] European Commission. "Towards a new Energy Strategy for Europe 2011-2020". ec.europa.eu/energy/strategies/consultations/doc/2010_07_02/2010_07_ 02_energy_strategy.pdf

[5] INTEGRIS: Intelligent electrical grid sensor communications [Online]. Available: www.fp7integris.eu

[6] L. Peretto, R. Tinarelli, A. Bauer, S. Pugliese, "Fault location in Underground Power Network: a Case Study", Proc. of the IEEE ISGT 2011, Anaheim, CA, USA, Jan. 17-19 2011, pp. 1-4.

[7] D. Della Giustina, S. Repo, S. Zanini and L. Cremaschini, "ICT Architecture for an Integrated Distribution Network Monitoring", accepted for publication on Proc. of the IEEE AMPS 2011, Aachen, Germany, Sep. 28-30 2011.

[8] S. Repo, D. Della Giustina, G. Ravera, L. Cremaschini, S. Zanini, J. M. Selga and P. Järventausta, "Use Case Analysis of Real-Time Low Voltage Network Management", accepted for publication on Proc. of the IEEE ISGT 2011, Manchester, UK, Dec. 5-7 2011. 